\title{
Relationship between ovarian and placental steroid production during early pregnancy in the marmoset monkey (Callithrix jacchus)
}

\author{
J. K. Hodges, Cilla Henderson and J. P. Hearn \\ Department of Reproduction, Institute of Zoology, The Zoological Society of London, Regent's Park, \\ London NWI 4RY, U.K.
}

\begin{abstract}
Summary. Concentrations of progesterone, $17 \alpha$-hydroxyprogesterone, oestrone and oestradiol-17 $\beta$ in peripheral and utero-ovarian vein blood were measured during the first 60 days of pregnancy. The same hormones were also measured in peripheral blood samples from non-fertile cycles. Peripheral levels of $17 \alpha$-hydroxyprogesterone, oestrone and oestradiol increased gradually during early pregnancy whereas concentrations of progesterone declined. The patterns of secretion of progesterone, $17 \alpha$-hydroxyprogesterone and oestrone, but not oestradiol, were significantly different in fertile and non-fertile cycles by 15 days after ovulation.

Comparison of hormone values in peripheral and utero-ovarian vein samples from ovaries with and without corpora lutea (Days 7, 9, 13, 21, 40 and 60 of pregnancy) showed that: (a) progesterone was secreted by the corpus luteum until at least Day 40 by which time there was also placental secretion; (2) although $17 \alpha$-hydroxyprogesterone was secreted by the corpus luteum, the relative contribution of luteal and placental secretion after Day 21 was not clear; (3) oestrone secretion by the corpus luteum was no longer detectable by Day 40, but placental oestrone secretion appeared to be present by this time; (4) the corpus luteum did not secrete significant amounts of oestradiol at any stage of early pregnancy, although there was evidence for placental secretion by Day 40 .
\end{abstract}

These results suggest that progesterone secretion by the corpus luteum of early pregnancy continues beyond the time when oestrogen secretion has declined. The corpus luteum to placental shift in the marmoset appears to occur at a later stage of pregnancy than it does in the macaque monkey and probably also in man.

\section{Introduction}

Sustained steroid secretion by the corpus luteum is thought to be required for the establishment and maintenance of early pregnancy in most primate species. As pregnancy proceeds, the placenta becomes the major source of steroids and the corpus luteum is dispensable. In women, the corpus luteum is necessary for the first 5-6 weeks of gestation (Csapo, Pulkkinen \& Wiest, 1973; Csapo \& Pulkkinen, 1978) whereas pregnancy in the rhesus monkey will continue in the absence of the corpus luteum after Day 21 (Tullner \& Hertz, 1966; Goodman \& Hodgen, 1979). There are, however, very few comparable data in other primates. Furthermore, with the possible exception of the rhesus monkey, details of luteal function during early pregnancy and the proportion of steroids secreted by the ovary and placenta before completion of the luteoplacental shift in primates have yet to be established. 
Peripheral steroid concentrations throughout pregnancy in the marmoset monkey have been reported (Chambers \& Hearn, 1979). Although there is evidence that the placenta is the major source of progesterone by Week 10 of pregnancy (Hearn \& Chambers, 1980), very little is known about the site of production of steroids before this time. The present paper therefore examines the secretion of steroids by the corpus luteum and placenta during the first 60 days of gestation in the marmoset to determine the relationship between ovarian and placental function before and during the luteoplacental shift in this primate.

\section{Materials and Methods}

Animals. Sexually mature female marmoset monkeys (body weight $0 \cdot 3-0 \cdot 4 \mathrm{~kg}$ ) from the Institute of Zoology primate colony were used. Details of animal management and housing conditions have been published (Hearn, Lunn, Burden \& Pilcher, 1975).

Sample collection. Blood samples were collected into a heparinized $1.0 \mathrm{ml}$ syringe and placed immediately on ice. Peripheral blood samples $(0.4 \mathrm{ml})$ were taken from the femoral vein without sedation. Samples $(0.2-0.4 \mathrm{ml})$ from the left and right utero-ovarian veins were collected simultaneously with peripheral samples and the number and distribution of corpora lutea were recorded during mid-line laparotomy under Saffan anaesthesia $(18 \mathrm{mg} / \mathrm{kg}$ i.m.; alphaxalone, $0.9 \%$, $\mathrm{w} / \mathrm{v}$ and alphadalone acetate, $0 \cdot 3 \% \mathrm{w} / \mathrm{v}$ : Glaxo Laboratories Ltd, Greenford, Middx, UB6 0HE, U.K.). Details of surgical procedures and collection of internal blood samples have been described (Hearn \& Chambers, 1980). The blood was centrifuged at $500 \mathrm{~g}$ for $20 \mathrm{~min}$ and the plasma stored at $-20^{\circ} \mathrm{C}$ until assayed.

The schedule for bleeding is indicated in Text-fig. 1. Post-partum females were initially bled 3 times a week and progesterone levels measured to detect ovulation. A rise in progesterone to values exceeding $10 \mathrm{ng} / \mathrm{ml}$ was taken to indicate the day after ovulation, i.e. Day 1 (Harlow, Gems, Hodges \& Hearn, 1983). The later stages of pregnancy ( $>20$ days) were confirmed by maintenance of elevated progesterone concentrations $(>30 \mathrm{ng} / \mathrm{ml})$ and by uterine palpation. Early stages of pregnancy ( $<20$ days) were confirmed retrospectively (as above) or by recovery of embryos after uterine flushing. Samples for non-fertile cycles were collected from females paired with castrated or vasectomized males.

Since laparotomy was performed once during individual pregnancies the utero-ovarian data are for non-repeated samples. Peripheral blood samples were collected sequentially from non-pregnant animals and for various periods from most pregnant animals.

Assays. All hormones were measured after celite column chromatography. Full details of the assays for oestrone and oestradiol-17 $\beta$ in marmoset plasma have been described (Hodges, Brand, Henderson \& Kelly, 1983a). Progesterone was measured according to the method described by Hodges, Eastman \& Jenkins (1983b), except that progesterone was eluted from a propylene glycol : celite $(1: 2 \mathrm{v} / \mathrm{w})$ column with $5.0 \mathrm{ml}$ iso-octane after a $0.5 \mathrm{ml}$ iso-octane rinse; the buffer used for assay was $0.01 \mathrm{M}$-phosphate buffer, containing $0.1 \%$ gelatin. The origins and cross-reactivities of the antisera have been reported (Hodges et al., 1983a, b).

The assay for $17 \alpha$-hydroxyprogesterone was based on the method described by Hodges et al. (1983a). Tracer amounts (1500 c.p.m.) of $\left[{ }^{3} \mathrm{H}\right] 17 \alpha$-hydroxyprogesterone (sp. act. $61 \mathrm{Ci} / \mathrm{mmol}$; Radiochemical Centre, Amersham, U.K.) were added to samples before extraction with redistilled diethyl ether. After evaporation with nitrogen, the residue was reconstituted in $1.0 \mathrm{ml}$ iso-octane ready for celite chromatography using the same column system as for progesterone: $5 \mathrm{ml} 5 \%$ and $10 \%$ ethyl acetate in iso-octane were used as additional rinses and $5 \mathrm{ml} 20 \%$ ethyl acetate in isooctane was used to elute $17 \alpha$-hydroxyprogesterone. The sample was dried under nitrogen and reconstituted in $1.0 \mathrm{ml}$ phosphate buffer for assay and recovery determination. The antiserum (Steranti Research Ltd, St Alban's, Herts, U.K.) was raised in a rabbit against $17 \alpha$-hydroxyprogesterone-3-(O-carboxymethyl)oxime-bovine serum albumin and showed the following cross- 
reactivities: progesterone $(1.0 \%), 17 \alpha-\mathrm{OH}$-pregnenolone $(0.5 \%), 5 \alpha$-pregnanedione $(0.5 \%), 20 \alpha-$ dihydroprogesterone $(0.1 \%)$, other $\mathrm{C}_{21}, \mathrm{C}_{19}$ and $\mathrm{C}_{18}$ steroids tested $(<0.1 \%)$. Validation of the measurement of progesterone and $17 \alpha$-hydroxyprogesterone was provided by the parallel profiles of radioactivity and immunoreactivity obtained for each hormone by celite co-chromatography (Hodges et al., 1983a). Procedural losses were monitored individually and mean \pm s.e. recovery values $(\%)$ for each assay were $65 \pm 5.0$ (oestrone), $75 \pm 5.4$ (oestradiol-17ß), $63 \pm 5.6$ (progesterone) and $67 \pm 5.6(17 \alpha$-hydroxyprogesterone). The sensitivity of each assay, defined as the least amount of hormone detectable (i.e. $90 \%$ binding) in marmoset plasma $(\mathrm{ng} / \mathrm{ml}$, peripheral and utero-ovarian vein plasma, respectively) was as follows: oestrone, $0.32,0.65$; oestradiol-17 $0 \cdot 15,0.37$; progesterone, $5 \cdot 7,22 \cdot 8 ; 17 \alpha$-hydroxyprogesterone, $2 \cdot 6,10 \cdot 4$. Values are different for peripheral and utero-ovarian vein samples because of the different dilutions required. Buffer blanks, extracted and run through celite in each assay, gave values less than the sensitivities quoted. Interassay coefficients of variation were between 8 and $13 \%$ based on replicate determinations after chromatography of a marmoset plasma pool.

Analysis of data. Data for peripheral samples were analysed (after logarithmic transformation) by the least square means procedure (Statistical Analysis System, 1979) using two-way and, where appropriate, one-way analysis of variance. Means were compared post hoc by Duncan's Multiple Range Test. Utero-ovarian vein data were analysed by one-way analysis of variance. Differences between peripheral and utero-ovarian vein hormone levels were evaluated by a Student's $t$ test and a Wilcoxon's rank sum test was used to compare hormone levels of utero-ovarian vein samples associated with 1 or 2 corpora lutea.

\section{Results}

\section{Peripheral hormone levels}

The concentrations of progesterone, $17 \alpha$-hydroxyprogesterone, oestrone and oestradiol- $17 \beta$ during early pregnancy and in the luteal phase of the non-fertile cycle are shown in Text-fig. 1. There was a gradual decline in progesterone concentrations during the first 60 days of pregnancy $(P<0.05)$ whereas levels in non-fertile cycles fell markedly between Days 11 and 15 . The progesterone profiles in pregnant and non-pregnant animals were significantly different by 15 days after ovulation $(P<0.01)$. However, levels of $17 \alpha$-hydroxyprogesterone increased during the same period of early pregnancy $(P<0.01)$. Therefore, during the first 21 days progesterone concentrations were double those of $17 \alpha$-hydroxyprogesterone whereas by Day 60 the ratio was $<1$ (the ratios at these times were significantly different, $P<0.05$, Student's $t$ test). Levels of $17 \alpha$-hydroxyprogesterone in pregnant and non-pregnant animals differed by Day 15 after ovulation $(P<0.02)$.

Oestrone and oestradiol-17 $\beta$ also increased significantly during the first 60 days of pregnancy $(P$ $<0.01, P<0.05$ respectively). Levels of oestradiol- $17 \beta$ were, however, considerably lower than those of oestrone, and in fertile and non-fertile cycles there was an oestrone :oestradiol-17 $\beta$ concentration ratio of $>5: 1$. There was a significant difference in oestrone profiles between fertile and non-fertile cycles by Day 15 after ovulation $(P<0.02)$, whereas no differences were found for levels of oestradiol-17ß $(P>0.05)$.

\section{Distribution of corpora lutea}

Laparotomy during early pregnancy was performed on 28 marmosets: 5, 19 and 4 marmosets had 1,2 or 3 corpora lutea respectively. In 13 of the 23 animals with more than one corpus luteum all visible corpora lutea were restricted to a single ovary. The utero-ovarian vein data are therefore derived from the vein ipsilateral to an ovary without a corpus luteum or from the vein ipsilateral to an ovary bearing one or more corpora lutea. The distribution of utero-ovarian vein samples in the latter group, according to the number of corpora lutea on the ovary, is shown in Table 1. All utero- 


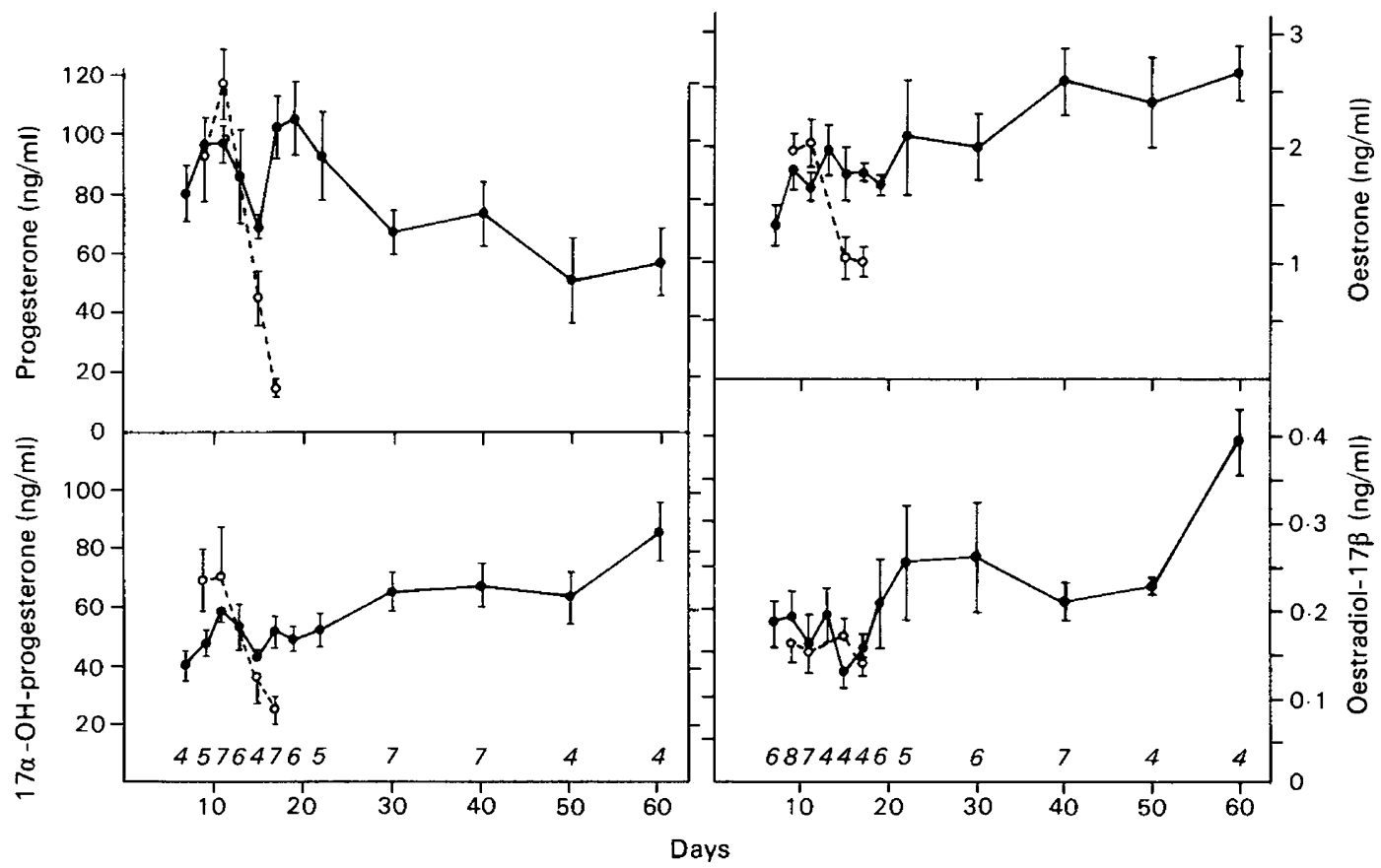

Text-fig. 1. Peripheral concentrations of progesterone, $17 \alpha$-hydroxyprogesterone, oestrone and oestradiol-17 $\beta$ in the luteal phase of the ovarian cycle and during the first 60 days of pregnancy in the marmoset. Data (mean \pm s.e.m.) are for Days $9,11,15$ and 17 after ovulation in the luteal phase $(O-O, N=4)$ and from Day 7 after ovulation during pregnancy $(O-O$, $\mathbf{N}=4-8$, as shown at each datum point).

Table 1. The number of individual samples collected from utero-ovarian veins draining ovaries with 1,2 or 3 corpora lutea at different stages of pregnancy in marmosets

\begin{tabular}{cccccccc}
\hline & \multicolumn{7}{c}{ Stage of pregnancy (days) } \\
\cline { 2 - 6 } $\begin{array}{c}\text { No. of } \\
\text { corpora lutea }\end{array}$ & 7 & 9 & 13 & 21 & 40 & 60 & Total \\
\hline 1 & 2 & 6 & 2 & 4 & 1 & 2 & 17 \\
2 & 4 & 3 & 2 & 3 & 3 & 3 & 18 \\
3 & 0 & 0 & 1 & 0 & 0 & 0 & 1 \\
Total * $^{*}$ & 6 & 9 & 5 & 7 & 4 & 5 & - \\
\hline
\end{tabular}

* The numbers in this row correspond to those shown in Text-fig. 2 (utero-ovarian vein with corpus luteum).

ovarian vein samples from ovaries bearing 1,2 , or 3 corpora lutea at each day of pregnancy are grouped together and the data reported as such in Text-fig. 2 . There was no significant difference in hormone concentrations of utero-ovarian vein samples associated with 1 or 2 corpora lutea $(P>$ 0.05 ), most probably due to the small sample number and large individual variation.

\section{Hormone concentrations in utero-ovarian vein plasma}

Concentrations of progesterone, $17 \alpha$-hydroxyprogesterone, oestrone and oestradiol-17 $\beta$ in peripheral and utero-ovarian vein plasma are compared at different stages of pregnancy in Text- 


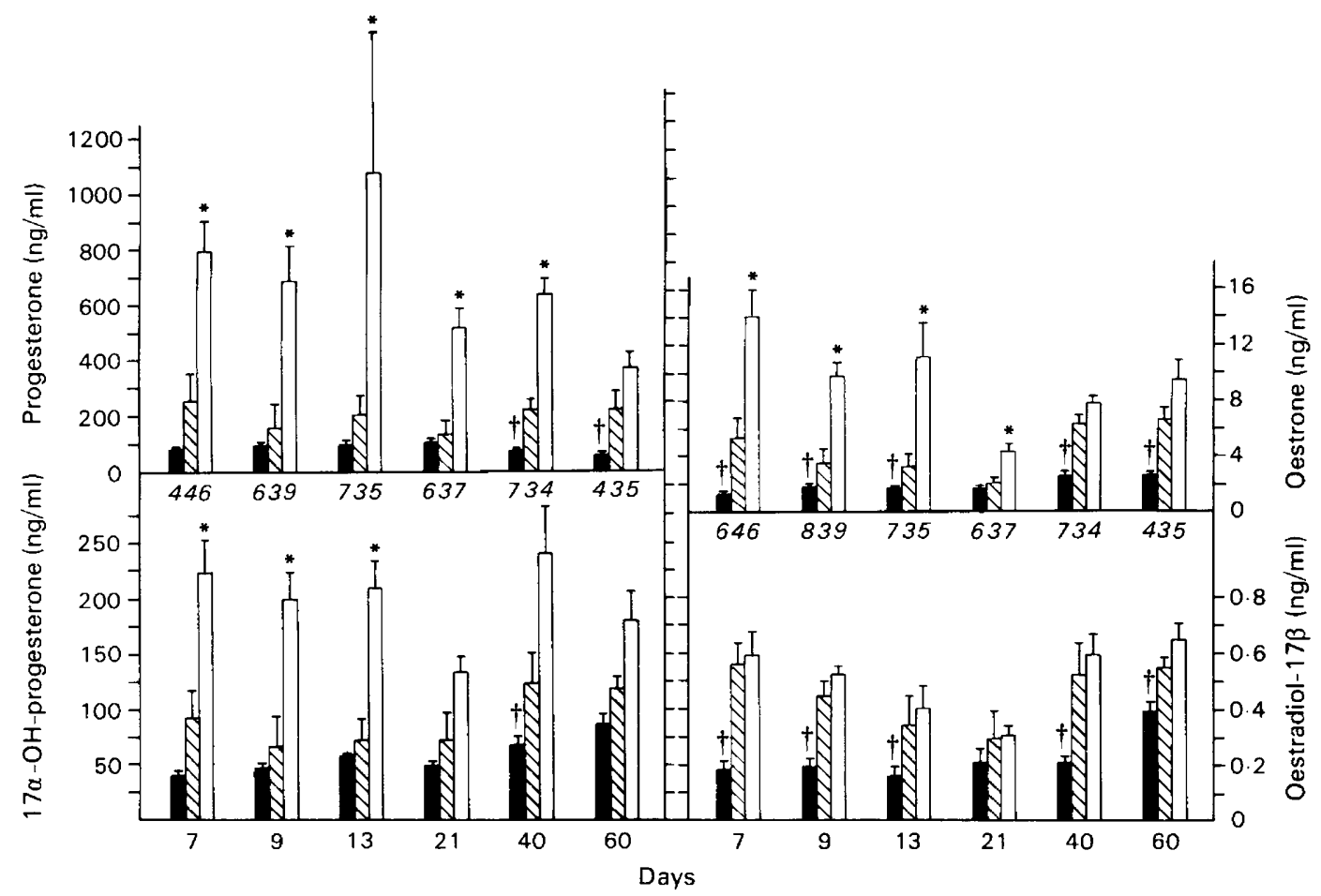

Text-fig. 2. Concentrations of progesterone, 17 $\alpha$-hydroxyprogesterone, oestrone and oestradiol- $17 \beta$ in the peripheral circulation $(\square)$ and in utero-ovarian veins draining ovaries with $(\square)$

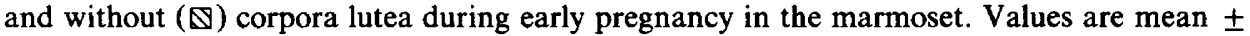
s.e.m. and the number of determinations in each category is shown. ${ }^{*} P<0.05-P<0.01$ for hormone levels in utero-ovarian veins with and without a corpus luteum. $\dagger P<0.05-P<0.01$ for levels in the utero-ovarian vein without a corpus luteum and levels in peripheral blood.

fig. 2. In all cases, except for oestradiol-17 $\beta$ on Day 21 , hormone concentrations in at least one utero-ovarian vein were higher than those in peripheral plasma $(P<0.05)$. There were no significant changes in the utero-ovarian vein concentrations of progesterone or $17 \alpha$-hydroxyprogesterone related to the stage of pregnancy $(P>0.05)$. Progesterone levels were higher in the vein associated with a corpus luteum up to Day 40 of pregnancy, but not at Day 60 . Progesterone levels in the utero-ovarian vein not associated with a corpus luteum were also higher than those in peripheral samples on Days 7-13 of pregnancy, but the differences were not significant until Day 40 (probably due to large individual variation as indicated by the standard error values). Concentrations of $17 \alpha$-hydroxyprogesterone were also higher in the utero-ovarian vein associated with a corpus luteum on Days 7-13. The only significant difference between $17 \alpha$-hydroxyprogesterone levels in the utero-ovarian vein not associated with a corpus luteum and peripheral levels was at Day 40 . The reversal of the progesterone : $17 \alpha$-hydroxyprogesterone concentration ratio in peripheral plasma was not evident in utero-ovarian vein plasma in which progesterone was consistently present in higher concentrations.

The concentrations of oestrone and oestradiol- $17 \beta$ in the utero-ovarian vein associated with a corpus luteum declined between 7 and 21 days of gestation but then increased to Day $60(P<0.01)$. There was a significant difference in oestrone levels between utero-ovarian veins at each stage of pregnancy until Day 21, but not at Days 40 or 60 . In contrast, however, there were no such differences between utero-ovarian oestradiol-17 $\beta$ concentrations at any stage of pregnancy. 
Differences in the levels of oestrone or oestradiol-17 $7 \beta$ between utero-ovarian vein and peripheral plasma were present on Days 7-13, 40 and 60 of pregnancy, but not on Day 21. As in peripheral blood, the levels of oestrone in the utero-ovarian vein were considerably higher than those of oestradiol-17 $\beta$.

\section{Discussion}

The gradual changes in the peripheral concentrations of progesterone, oestrone and oestradiol$17 \beta$ during the first 60 days of pregnancy in the marmoset show more similarities to those in women (Tulchinsky \& Hobel, 1973; Mishell, Thorneycroft, Nagata, Murata \& Nakamura, 1973; Corker, Michie, Hobson \& Parboosingh, 1976) and chimpanzees (Reyes, Winter, Faiman \& Hobson, 1975) than to the more abrupt changes that occur in the rhesus monkey (Atkinson et al., 1975). The pattern of increasing $17 \alpha$-hydroxyprogesterone concentrations, however, differs from that in human pregnancy in which there is a gradual fall in levels between the 4th and 6th week of gestation (e.g. Mishell et al., 1973).

Differences between the patterns of progesterone, 17 $\alpha$-hydroxyprogesterone and oestrone secretion in pregnant and non pregnant marmosets appear to occur between 12 and 15 days after ovulation. This is later than reported in other primate species such as women (Lenton, Sulaiman, Sobowale \& Cooke, 1982), chimpanzees (Reyes et al., 1975), and rhesus monkeys (Atkinson et al., 1975) in which oestrogen and/or progesterone levels are higher in fertile than in non-fertile cycles by 10-12 days after ovulation. This species difference probably relates to the longer luteal phase of 19-20 days in the marmoset (Harding, Hulme, Lunn, Henderson \& Aitken, 1982; Harlow et al., 1983) compared with an average luteal phase duration of 14-16 days in women (e.g. Lenton et al., 1982) and most other non-human primates (see Hendrickx \& Enders, 1980, for references). The reason for the extended luteal phase in the marmoset is not known, but the possible relationship between the length of the luteal phase and the time of implantation and onset of chorionic gonadotrophin secretion in this primate is currently being examined.

Utero-ovarian vein samples were collected in an attempt to examine the relative contribution of the corpus luteum and placenta to circulating steroid levels during early pregnancy. However, since comparable data from non-pregnant animals are not available, the findings reported for Days 7-13 may not be specific to pregnancy and similar changes may also occur during the luteal phase of the non-fertile cycle. Comparison of peripheral and utero-ovarian vein progesterone concentrations shows that the marmoset corpus luteum secretes progesterone at Day 40 of pregnancy but not at Day 60, and that there is placental secretion at both of these times. There is therefore a period, around Day 40, during which the ovarian and placental sources contribute to circulating progesterone levels. The decline in peripheral progesterone concentrations between 21 and 60 days of gestation probably reflects the inability of the increasing placental contribution to compensate for the decrease in luteal secretion.

The decline in luteal progesterone production and the onset of placental secretion in the marmoset occurs at a later stage of pregnancy than in macaque monkeys and in women when the much shorter gestation length of the marmoset is taken into consideration. In rhesus and cynomolgus monkeys, peripheral progesterone levels remain elevated after lutectomy on Day 23 of pregnancy (Williams, Johnson \& Hodgen, 1978). Measurement of progesterone in uterine and utero-ovarian vein samples indicated either no luteal secretion at this stage (Sholl, Anderson, Colas \& Wolf, 1977) or, more likely, a transitional period for the corpus luteum and placenta with regard to progesterone secretion (Walsh, Wolf \& Meyer, 1974). Regardless of the relative activity of the corpus luteum at this time, the placenta in the rhesus monkey clearly develops the ability to secrete progesterone at an early stage since peripheral progesterone levels can increase between Days 22 and 25 of pregnancy after bilateral ovariectomy at Day 17 (Goodman \& Hodgen, 1979). Studies involving removal of the corpus luteum of women have indicated a decline in luteal function and increasing placental contribution to progesterone levels after the 4th and 5 th week of gestation, 
although the corpus luteum is not completely dispensable until the 6th week (Csapo \& Pulkkinen, 1978).

During early pregnancy the human placenta lacks the $17 \alpha$-hydroxylase enzyme (Palmar, Blair, Eriksson \& Diczfalusy, 1966) and measurements of peripheral concentrations of $17 \alpha$-hydroxyprogesterone have been used by several investigators to document the decline in luteal steroid secretion that begins at 3 or 4 weeks of gestation (e.g. Tulchinsky \& Hobel, 1973). No decline in peripheral levels of $17 \alpha$-hydroxyprogesterone up to 60 days of pregnancy was noted in the present study. The lack of significant differences between utero-ovarian vein concentrations makes it difficult to determine the origin of the $17 \alpha$-hydroxyprogesterone after 21 days of pregnancy. However, an increase in peripheral concentrations in the absence of any increase in utero-ovarian vein levels suggests that there may be a change in peripheral metabolism of $17 \alpha$-hydroxyprogesterone, although changes in the blood flow in the internal vessels might also be important (Lees, Hill, Ochsner, Thomas \& Novy, 1971). The reversal of the progesterone : $17 \alpha$-hydroxyprogesterone concentration ratio from Days 11 to 40 in peripheral but not in utero-ovarian blood lends support to the involvement of changes in hormone metabolism. Placental secretion of $17 \alpha$-hydroxyprogesterone, should it occur in the marmoset, might not be unusual since Walsh et al. (1974) concluded that circulating $17 \alpha$-hydroxyprogesterone levels were predominantly of placental origin at Day 42 of pregnancy in the rhesus monkey.

Oestrone secretion by the marmoset corpus luteum was no longer detectable by Day 40 of gestation, whereas both oestrone and oestradiol-17 $\beta$ appeared to be secreted by the placenta at this time. There was, however, a decline in utero-ovarian vein levels of oestrone and oestradiol-17 between Days 7 and 21, suggesting that ovarian oestrogen secretion was already decreasing over this period. It therefore appears that the marmoset corpus luteum retains the ability to secrete progesterone beyond the time when oestrogen (oestrone) secretion has ceased. The increase in utero-ovarian levels of oestrone and oestradiol-17 $\beta$ between Day 20 and 60 of gestation is interpreted to reflect increasing placental secretion.

In contrast to oestrone, oestradiol-17 $\beta$ was not secreted in detectable amounts by the corpus luteum at any stage of early pregnancy, although there was clearly an ovarian contribution between Days 7 and 13. Therefore, before Day 21 of pregnancy, oestradiol-17 $\beta$ appears to derive from nonluteal ovarian tissue, whereas oestrone is secreted by luteal and non-luteal compartments, but predominantly by the corpus luteum. Since oestradiol-17 $\beta$ is secreted in abundance by the corpus luteum of non-pregnant (Stouffer, Bennett \& Hodgen, 1980) and early pregnant (Walsh, Wolf, Meyer \& Robinson, 1979) rhesus monkeys and is the major luteal oestrogen in non-pregnant women (Swanston, McNatty \& Baird, 1977), the inability to demonstrate oestradiol-17 $\beta$ secretion by the corpus luteum in the marmoset is unusual.

Studies of rhesus monkeys by Williams et al. (1978) and Walsh et al. (1979) have shown that the ovary bearing the corpus luteum and the placenta both secrete oestrone and oestradiol-17 $\beta$ on Day 22 of pregnancy. By Day 42, however, there is no longer any substantial ovarian contribution and virtually all circulating oestrogens originate from the placenta (Walsh et al., 1979). Few comparable data exist for women. Rao (1979) has demonstrated in-vitro synthesis of oestradiol-17 $\beta$ and progesterone from endogenous precursors in the human placenta from at least 5 weeks gestation, although completion of placental takeover in hormone production does not occur until closer to 7 weeks.

These results demonstrate that during early pregnancy in the marmoset the corpus luteum continues to secrete significant amounts of progesterone until at least Day 40, whereas oestrone secretion is no longer evident at this time. The absence of detectable oestradiol-17 $\beta$ secretion by the corpus luteum at any stage is of interest although the significance of this in terms of ovarian function in this primate is not yet clear. The placenta secretes increasing amounts of oestrogens and progesterone from about Day 40 and probably accounts for the major proportion of steroid secretion by Day 60 . Thus, in relation to total gestation length the luteo-placental shift occurs somewhat later in the marmoset than in the macaque monkey and in man. 
We thank Mrs B. Murrill and the Animal House staff for care and maintenance of the marmosets; Miss S. Gems for assistance with blood sampling; Mrs D. Bevan for help with progesterone assays; Dr D. H. Abbott for help with statistical analysis; and the World Health Organization for antisera and standards for the progesterone and oestradiol-17 $\beta$ assays. The work was supported by an M.R.C. programme grant to J.P.H. and by a core support grant from the A.B.R.C. to the Institute of Zoology.

\section{References}

Atkinson, L.E., Hotchkiss, J., Fritz, G.R., Surve, A.H., Neill, J.D. \& Knobil, E. (1975) Circulating levels of steroids and chorionic gonadotropin during pregnancy in the rhesus monkey, with special attention to the rescue of the corpus luteum in early pregnancy. Biol. Reprod. 12, 335-345.

Chambers, P.L. \& Heam, J.P. (1979) Peripheral plasma levels of progesterone, oestradiol- $17 \beta$, oestrone, testosterone, androstenedione and chorionic gonadotrophin during pregnancy in the marmoset monkey (Callithrix jacchus). J. Reprod. Fert. 56, 23-32.

Corker, C.S., Michie, E., Hobson, R. \& Parboosingh, J. (1976) Hormonal patterns in conceptual cycles and early pregnancy. Br. J. Obstet. Gynaec. 83, 489-494.

Csapo, A.I. \& Pulkkinen, M.O. (1978) Indispensability of the human corpus luteum in the maintenance of early pregnancy: lutectomy evidence. Obstet. Gynaec. Surv. 33, 69-80.

Csapo, A.I., Pulkkinen, M.O. \& Wiest, W.G. (1973) Effects of lutectomy and progesterone replacement in early pregnant patients. Am. J. Obstet. Gynec. 115, 759-765.

Goodman, A.J. \& Hodgen, G.D. (1979) Corpus luteumconceptus-follicle relationships during the fertile cycle in rhesus monkeys: pregnancy maintenance despite early luteal removal. J. clin. Endocr. Metab. 49, $469-471$.

Harding, R.D., Hulme, M.J., Lunn, S.F., Henderson, C. \& Aitken, J. (1982) Plasma progesterone levels throughout the ovarian cycle of the common marmoset (Callithrix jacchus). J. med. Primatol. 11, 43-51.

Harlow, C.R., Gems, S., Hodges, J.K. \& Hearn, J.P. (1983) The relationship between plasma progesterone and the timing of ovulation and early embryonic development in the marmoset monkey. J. Zool., Lond. (in press).

Hearn, J.P. \& Chambers, P.L. (1980) Progesterone secretion by the ovary and placenta during pregnancy in the marmoset monkey (Callithrix jacchus). In NonHuman Primate Models for Study of Reproduction, pp. 82-87. Ed. T. C. Anand Kumar. Karger, Basel.

Hearn, J.P., Lunn, S.F., Burden, F.J. \& Pilcher, M.M. (1975) Management of marmosets for biomedical research. Lab. Anim. 9, 125-134.

Hendrickx, A.G. \& Enders, A.C. (1980) Implantation in non-human primates. II. Endocrinology. In NonHuman Primate Models for Study of Reproduction, pp. 109-115. Ed. T. C. Anand Kumar. Karger, Basel.

Hodges, J.K., Brand, H.M., Henderson, C. \& Kelly, R.W. (1983a) The levels of circulating and urinary oestrogens during pregnancy in the marmoset monkey (Callithrix jacchus). J. Reprod. Fert. 67, 73-82.
Hodges, J.K., Eastman, S.A.K. \& Jenkins, N. (1983b) Sex steroids and their relationship to binding proteins in the serum of the marmoset monkey. J. Endocr. 96, 443-460.

Lees, M.H., Hill, J.D., Ochsner, A.J., Thomas, C.L. \& Novy, M.J. (1971) Maternal placental and myometrial blood flow of the rhesus monkey during uterine contractions. Am. J. Obstet. Gynec. 110, 68-81.

Lenton, E.A., Sulaiman, R., Sobowale, O. \& Cooke, I.D. (1982) The human menstrual cycle: plasma concentrations of prolactin, LH, FSH, oestradiol and progesterone in conceiving and non-conceiving women. J. Reprod. Fert. 65, 131-139.

Mishell, D.R., Thomeycroft, I.H., Nagata, Y., Murata, T. \& Nakamura, R.M. (1973) Serum gonadotropin and steroid patterns in early human gestation. Am. J. Obstet. Gynaec. 117, 631-639.

Palmar, R., Blair, J.A., Eriksson, G. \& Diczfalusy, E. (1966) Metabolism of progesterone and $20 \alpha$ and $20 \beta$ dihydroprogesterone by mid term placentas perfused in situ. Acta endocr., Copenh. 53, 407-414.

Rao, B. (1979) Effect of prostaglandins on in vitro synthesis of progesterone and estradiol-17 $\beta$ in placenta from early human pregnancy. Prostaglandins 18, 93-99.

Reyes, F.I., Winter, J.S.D., Faiman, C. \& Hobson, W.C. (1975) Serial serum levels of gonadotropins, prolactin and sex steroids in the non-pregnant and pregnant chimpanzee. Endocrinology 96, 1447-1455.

Sholl, S.A., Anderson, N.G., Colas, A.E. \& Wolf, R.C. (1977) Placental and luteal steroidogenesis in the pregnant rhesus monkey. Sterqids 29, 249-259.

Stouffer, R.L., Bennett, L.A. \& Hodgen, G.D. (1980) Estrogen production by luteal cells isolated from rhesus monkeys during the menstrual cycle: correlation with spontaneous luteolysis. Endocrinology 106, 519--525.

Swanston, I.A., McNatty, K.P. \& Baird, D.T. (1977) Concentrations of prostaglandin $F_{2} \alpha$ and steroids in the human corpus luteum. $J$. Endocr. 73, 115-122.

Tulchinsky, D. \& Hobel, C.J. (1973) Plasma human chorionic gonadotropin, estrone, estradiol, estriol, progesterone and $17 \alpha$-dihydroxyprogesterone in human pregnancy. III. Early normal pregnancy. Am. J. Obstet. Gynec. 117, 884-893.

Tullner, W.W. \& Hertz, R. (1966) Normal gestation and chorionic gonadotropin levels in the monkey after ovariectomy in early pregnancy. Endocrinology 78, 1076-1081

Walsh, S.W., Wolf, R.C. \& Meyer, R.K. (1974) Progesterone, progestins, and 17-hydroxypregn-4-ene-3,20dione in the utero-ovarian, uterine and peripheral 
blood of the pregnant rhesus monkey. Endocrinology 95, $1704-1710$.

Walsh, S.W., Wolf, R.C., Meyer, R.K. \& Robinson, J.A. (1979) Estrogens in the utero-ovarian, uterine and peripheral plasma in pregnant rhesus monkeys. Biol. Reprod. 20, 606-610.
Williams, R.F., Johnson, D.K. \& Hodgen, G.D. (1978) Ovarian estradiol secretion during early pregnancy in monkeys: luteal versus extra-luteal secretions and effect of chorionic gonadotropin. Steroids 32, 539545.

Received 9 March 1983 